\title{
The effect of grain size on dynamic tensile extrusion behaviour
}

\author{
Leeju Park ${ }^{\mathrm{a}}$, Hack Jun Kim, and Seok Bong Kim \\ Agency for Defense Development, PO Box 35-42, Taejon 305-600, Korea
}

\begin{abstract}
Dynamic tensile extrusion (DTE) tests were conducted on coarse grained and ultrafine grained (UFG) OFHC Cu, Interstitial free (IF) Steel, and pure Ta. Equal channel angular pressing (ECAP) of 16passes with $\mathrm{Bc}$ for $\mathrm{Cu}$, IF Steel and 4 passes for Ta was employed to fabricated UFG materials. DTE tests were carried out by launching the sphere samples (Dia. $7.62 \mathrm{~mm}$ ) to the conical extrusion die at a speed of $\sim 500 \mathrm{~m} / \mathrm{sec}$. The fragmentation behavior of the soft-recovered fragments were examined and compared with each other. The DTE fragmentation behavior of CG and UFG was numerically simulated by the LS-DYNA FEM code.
\end{abstract}

\section{Introduction}

DTE technique is a newly developed mechanical test [1]. In the ordinary DTE test, a spherical sample launched at high velocity passes through an open conical die. Due to the smaller die exit diameter than the sample diameter, the sample experiences severe tensile deformation. Therefore, the DTE test can characterize the mechanical response of materials under both high strain rate and high strain circumstances. The DTE technique has been applied to coarse grained (CG) pure metals such as $\mathrm{Cu}[1]$, Ta [2], $\mathrm{Zr}$ [3], etc..

Extensive and intensive researches during past two decades clearly reveal that ultrafine grained (UFG) materials exhibit very different mechanical and thermal responses from CG materials. There are several studies on mechanical behavior of UFG materials at high strain rates [4]. However, the strain rate employed in those studies (typically $10^{3} \mathrm{~s}^{-1}$ order) was quite lower than that being attainable in the dynamic tensile extrusion (DTE) test.

Ultrafine grained (UFG) materials usually exhibit higher strength due to the Hall-Petch strengthening but lower ductility due to shear localization than coarse grained (CG) counterparts at room temperature [5]. Meanwhile, some UFG materials show high strain rate super plasticity (HSRS) at high temperatures [6]. The shear localization is beneficial for the self-sharpening of the kinetic energy penetrator. HSRS is possible to operate on the metal jet formation of the metal liner in the chemical energy penetrator [7]. Accordingly, UFG materials are promising as the high performance penetrator materials. In this study, the metal jet formability of UFG OFHC $\mathrm{Cu}$, IF steel and pure Ta were compared to those of CG counterparts by means of DTE test.

\section{Experimental}

Commercial OFHC annealed $\mathrm{Cu}$ and IF steel bars $(18 \mathrm{~mm}$ diameter) were subjected to 16 passes equal channel

${ }^{a}$ Corresponding author: 1jparkr@add.re.kr angular pressing (ECAP) with route $\mathrm{B}_{\mathrm{c}}$ in order to fabricate equiaxed UFG samples. Pure Ta bars were conducted with 4 passes ECAP with route $B_{c}$ for fabricating UFG samples. The sphere samples of $7.62 \mathrm{~mm}$ diameter were machined from the central part of unECAPed and ECAPed bars for DTE tests. DTE tests were carried out by using an allvacuumed gas gun system which consists of the gas gun, the sample flying barrel, the DTE die chamber, and the sample recovery station; the details of the DTE equipment are described elsewhere [8]. The velocity of sample in this experiment was $\sim 500 \mathrm{~m} / \mathrm{sec}$ upon reaching the DTE die. After DTE tests, the sample fragments were soft recovered. The numbers and the order of fragments exiting the die were confirmed by the high speed photography. Besides, the complete fragment recovery was ensured by comparing the weight of all fragments with that of the initial sample. A routine microstructural observation were made on CG and UFG materials before the DTE tests with optical microscopy and electron backscattered diffraction (EBSD). The DTE behavior of CG and UFG $\mathrm{Cu}$ was numerically analyzed by using a commercial finite element code (LS-DYNA 2-dimensional axis-symmetric model [9]). The Johnson-Cook model was employed in the numerical analysis. Five unknown parameters in the Johnson-Cook model were obtained by conducting tensile tests at $10^{3} \mathrm{~s}^{-1}$ and $1 \mathrm{~s}^{-1}$ and compression tests at 2000 4000 s ${ }^{-1}$ on CG and UFG materials; tensile tests and compression tests were carried out on a hydraulic universal testing machine and a split Hopkinson pressure bar tester, respectively. In the numerial simulation process, the 2D R-adaptive remeshing was done in order to prevent severe distortion of the mesh. That is, a completely new mesh was created every $1 \mu \mathrm{sec}$ in order for the elements to keep a regular shape and a characteristic dimension. The new mesh is initialized from the old mesh by a least square approximation. The simulation results were compared with the experimetal ones in terms of total DTE elongation (sum of the axial elongation of individual fragment) and the number and the dimension of fragments). 


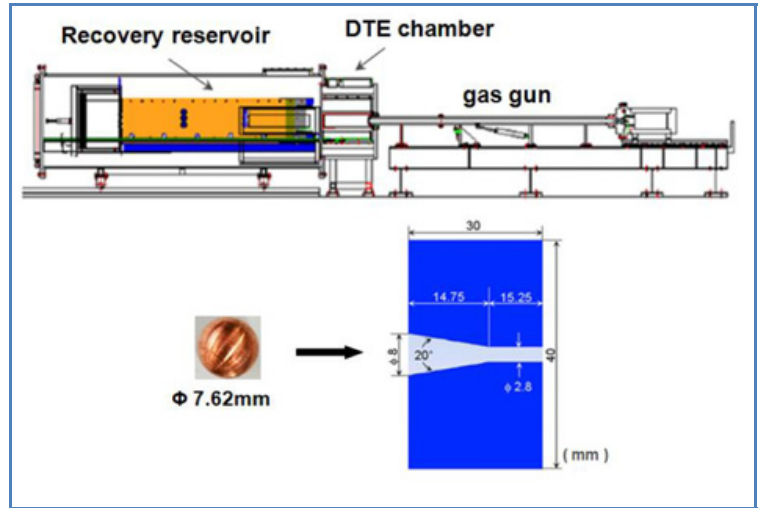

Figure 1. (a) The schematic illustration of the DTE facility consists of gas gun system, (b) Configuration of the DTE die (dimension in $\mathrm{mm}$ ).
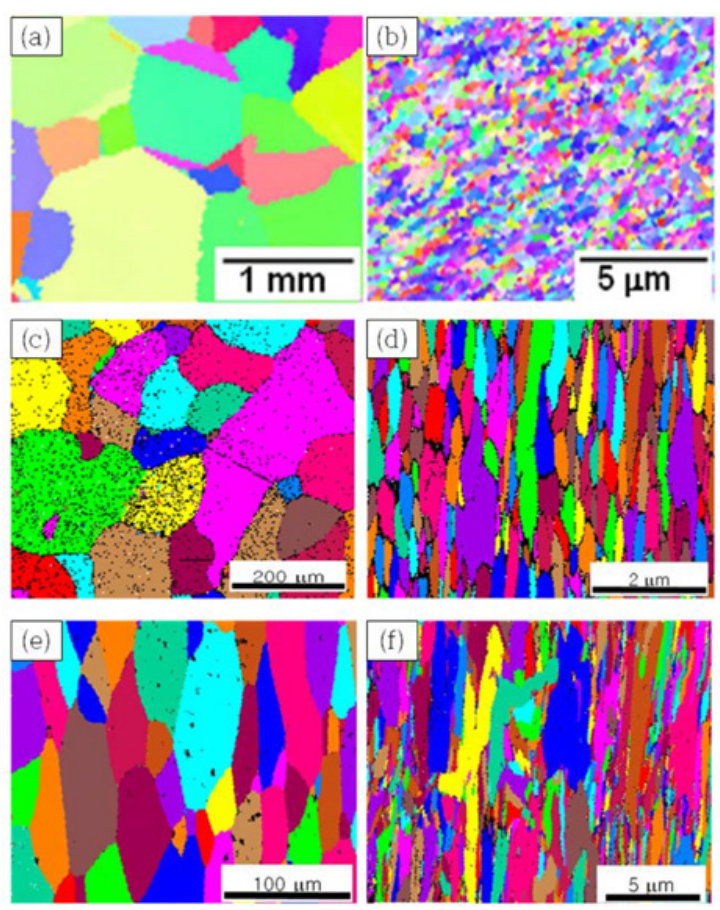

Figure 2. IPF maps (a) CG OFHC Cu, (b) UFG Cu, (c) CG IF Steel, (d) UFG IF Steel, (e) CG Ta, and (f) UFG Ta.

\section{Results and discussion}

\subsection{Examine the grain size of CG and UFG materials}

The Inverse pole figure (IPF) maps of the plane parallel to the extrusion axis of the CG and UFG samples are shown in Fig. 2. The average grain size of $\mathrm{CG}$ OFHC $\mathrm{Cu}$ was $\sim 1 \mathrm{~mm}$ and that of UFG $\mathrm{Cu}$ was $\sim 0.35 \mu \mathrm{m}$. The average grain size of IF steel was $\sim 163 \mu \mathrm{m}$ and that of UFG IF was $\sim 0.61 \mu \mathrm{m}$. The average grain size of CG Ta was $\sim 53 \mu \mathrm{m}$ and that of UFG Ta was $\sim 3.94 \mu \mathrm{m}$. IF steel and Ta has a weak texture after ECAP. The grain size difference of CG and UFG samples were range from a few times to $10^{6}$ times.
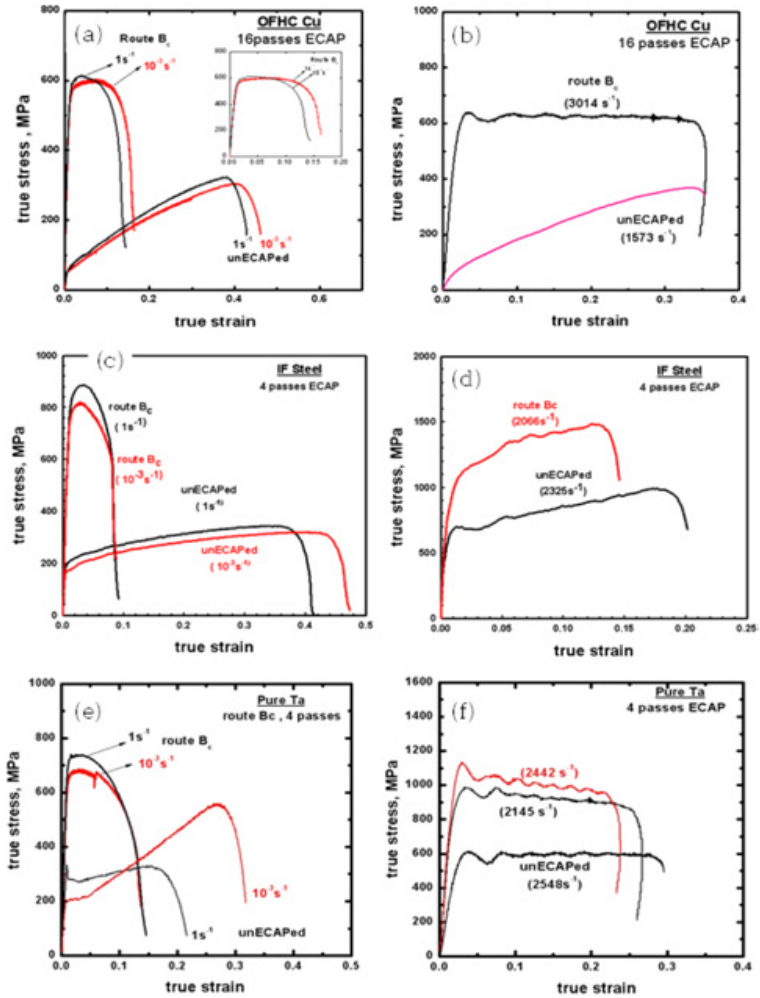

Figure 3. True stress-strain curves of (a) OFHC $\mathrm{Cu}$ at static, (b) OFHC Cu at dynamic, (c) IF steel at static, (d) IF Steel at dynamic, (e) Ta at static and (f) Ta at dynamic.

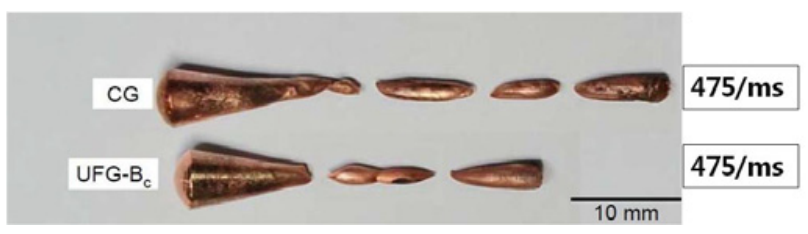

Figure 4. DTE fragments of $\mathrm{CG} \mathrm{Cu}$ and UFG OFHC $\mathrm{Cu}$ in sequence exiting the DTE die. The DTE direction is from left to right.

\subsection{Mechanical properties of CG and UFG materials}

The true stress-strain curves of CG and UFG sample are shown in Fig. 3. The yield stress of UFG materials were increased a few ten times that of CG materials. Meanwhile, the elongation of UFG materials were decreased a few times that of CG materials. As usual, regardless of the strain rate, $\mathrm{CG} \mathrm{Cu}$ and IF steel exhibited extensive strain hardening after low stress yielding while nearperfect plasticity without strain hardening after high stress yielding occurred in UFG $\mathrm{Cu}$ and Ta. While UFG IF steel showed strain hardening at high strain rate test $(\sim 2000 /$ s $)$ with SHPB.

\subsection{DTE behaviour: Fragmentation, ductility}

The representative soft-recovered fragments of the $\mathrm{CG} \mathrm{Cu}$ and UFG $\mathrm{Cu}$ after DTE are shown in Fig. 4: the conical fragment is the remnants remained in the DTE die. For all three runs of each sample, the $\mathrm{CG} \mathrm{Cu}$ was fragmented 


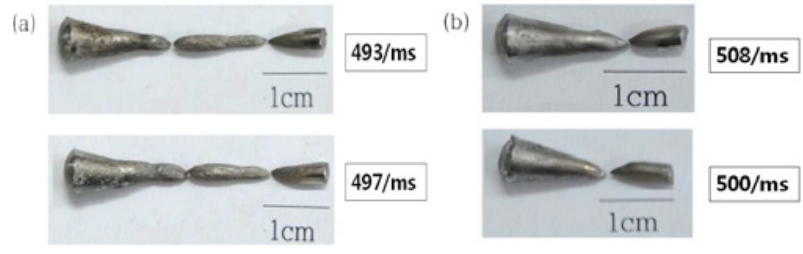

Figure 5. DTE fragments of (a) CG IF steel and (b) UFG IF steel in sequence exiting the DTE die.

(a)

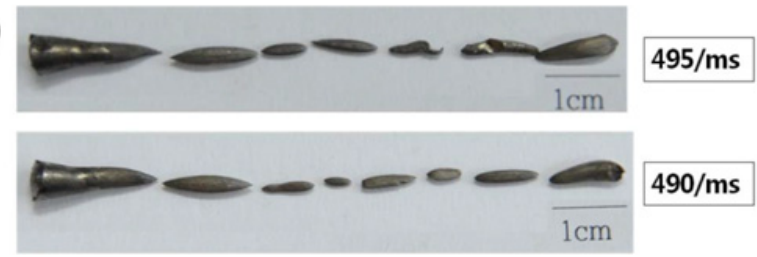

(b)

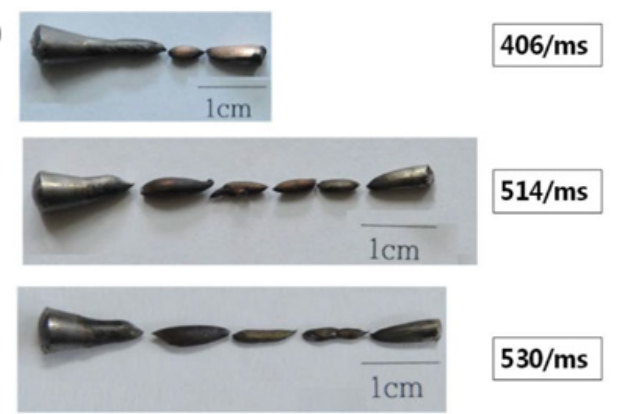

Figure 6. DTE fragments of CG and UFG pure Ta in sequence exiting the DTE die.

into 4 pieces while the UFG $\mathrm{Cu}$ was fragmented into 3 pieces. All fragments except the conical remnants were in the lenticular shape indicating that fragmentation occurred by plastic instability (i.e. necking) rather than void coalescence.

The average DTE ductility of each sample were $45.09 \mathrm{~mm}(\sim 592 \%)$ and $33.331 \mathrm{~mm}(\sim 437 \%)$ for the CG and UFG $\mathrm{Cu}$, respectively: the DTE ductility is the sum of the axial ductility of each fragment with respect to the initial sample diameter, i.e. DTE ductility $=\left(\mathrm{Sd}_{\mathrm{i}}-\mathrm{d}_{\mathrm{o}}\right) / \mathrm{d}_{\mathrm{o}}$ where $d_{i}$ is the longitudinal length of the $i^{\text {th }}$ fragment and $d_{o}$ is the initial sample diameter. The soft-recovered fragments of the CG and UFG IF Steel after DTE are shown in Fig. 5: the conical fragment is the remnants remained in the DTE die. For all two runs of each sample, the CG IF steel was fragmented into 3 pieces while the UFG IF Steel was fragmented into 1 piece. All fragments except the conical remnants were in the lenticular shape similar to OFHC $\mathrm{Cu}$. The DTE ductility of each sample was $41.31 \mathrm{~mm}(542 \%), 41.31 \mathrm{~mm}(518.8 \%)$ and $23.22 \mathrm{~mm}(\sim 305 \%), 23.16 \mathrm{~mm}(\sim 304 \%)$ for the CG and UFG IF steel, respectively.

The recovered fragments of the CG and UFG pure Ta after DTE are shown in Fig. 6. The CG tantalums were fragmented into 6 or 7 pieces while the UFG tantalums were fragmented into 4 or 5 piece. All fragments except the conical remnants were in the lenticular shape similar to the other above samples. The DTE ductility of each sample was $70.71 \mathrm{~mm}(\sim 930 \%), 72.2 \mathrm{~mm}(\sim 947.5 \%)$ for the CG

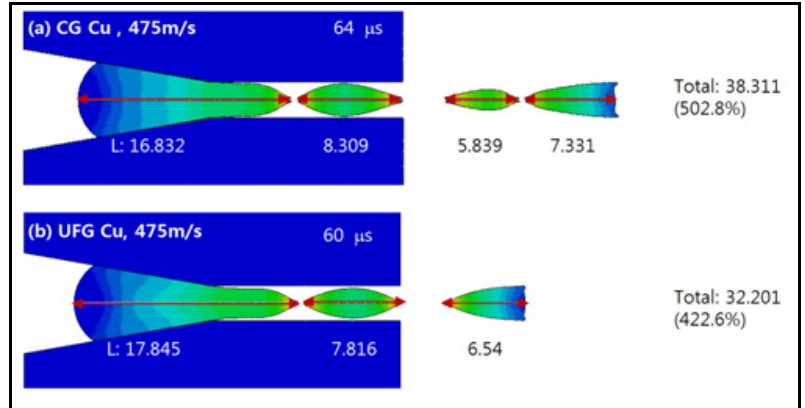

Figure 7. FEM simulation result showing the fragmentation of the DTE fragment of (a) CG Cu, and (b) UFG Cu.

and $33.51 \mathrm{~mm}(\sim 440 \%), 51.82 \mathrm{~mm}(680.05 \%), 49.35 \mathrm{~mm}$ $(\sim 647.6 \%) \%$ for the UFG tantalums, respectively.

\subsection{Numerical simulation results}

The present DTE behavior of CG and UFG OFHC $\mathrm{Cu}$ was numerically simlulated by the LS-DYNA FEM code with the 2D R-adaptivity remeshing. A simulation example for strain after complete fragmentation at $\sim 60 \mu \mathrm{sec}$ is presented in Fig. 7. The number of fragment is correctly prediced by simulation, i.e. 4 fragments for $\mathrm{CG} \mathrm{Cu}$ and 3 fragments for UFG $\mathrm{Cu}$. The simulated total length of $\mathrm{CG} \mathrm{Cu}$ and UFG $\mathrm{Cu}$ was $38.3 \mathrm{~mm}$ (DTE elongation $\sim 503 \%$ ) and $32.2 \mathrm{~mm}$ (DTE elongation $\sim 423 \%$ ), respectively. The simulated DTE elonagtion of UFG $\mathrm{Cu}$ is in reasonable agreement with the experimental one $(\sim 437 \%)$. In contrast, for $\mathrm{CG} \mathrm{Cu}$, the experimental DTE elongation $(\sim 592 \%)$ was larger than the simulated one $(503 \%)$. The maximum strain was developed at the necked region in both sample with the similar value. The strain at the necked region upon fragmentation reached $\sim 5.5$. Simulation revealed more localized necking in UFG $\mathrm{Cu}$, possibly causing smaller DTE elongation than $\mathrm{CG} \mathrm{Cu}$. In both sample, the strain rate was also maximum at the necked region with $10^{5} \mathrm{~s}^{-1}$ order which is at least one order or more higher than that achievable by the ordinary Hopkinson test. The maximum strain rate of UFG $\mathrm{Cu}$ was slightly higher than that of $\mathrm{CG} \mathrm{Cu}$, corresponding to more diffused strain distribution in the latter. The stress imposed by impacting the die was higher in UFG $\mathrm{Cu}$ due to its higher yield and flow stresses. The samle velocity was maximum at the exiting tips by the inertia effect. The tip (i.e. maximum) velocity of $\mathrm{CG} \mathrm{Cu}$ was faster than that of UFG $\mathrm{Cu}$. As expected considerable temperature rise occurred by adiabatic heating. Temperature at the stretched portion in the straight channel was close to or even higher than $700^{\circ} \mathrm{K}$ which is about $0.5 \mathrm{~T}_{\mathrm{m}}$. It was locally over $800^{\circ} \mathrm{K}\left(\sim 0.6 \mathrm{~T}_{\mathrm{m}}\right.$ of $\left.\mathrm{Cu}\right)$ upon fragmentation.

A simulation example DTE behavior of CG and UFG IF steeels were shown in Fig. 8. The number of fragment is correctly prediced by simulation, i.e. 3 fragments for CG steel and 2 fragments for UFG steel. The simulated total length of CG steel and UFG steel was $34.92 \mathrm{~mm}$ (DTE elongation 458\%) and $27.17 \mathrm{~mm}$ (DTE elongation $\sim 357 \%$ respectively. The simulated DTE elonagtion of CG and UFG steel is in reasonably well agreement with the experimental one $\sim 519 \%$ for CG and $\sim 304 \%$ for UFG. 


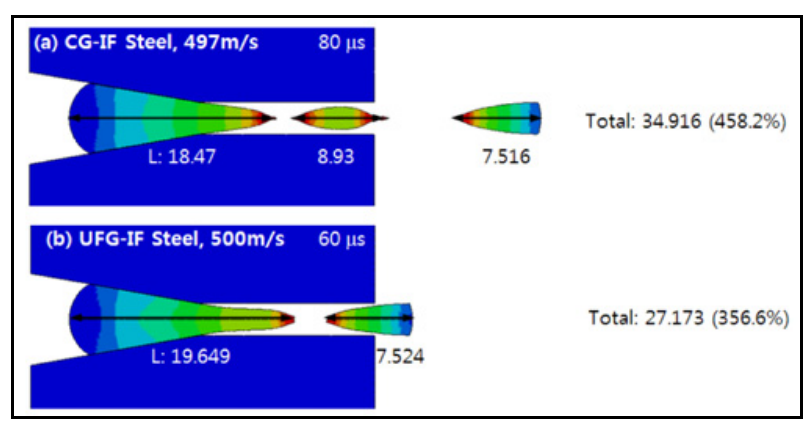

Figure 8. FEM simulation result showing the fragmentation of the DTE fragment of (a) CG IF steel, and (b) UFG IF steel.

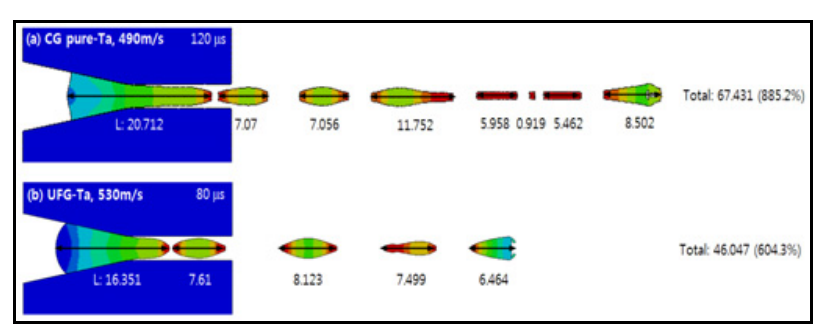

Figure 9. FEM simulation result showing the fragmentation of the DTE fragment of (a) CG Ta, and (b) UFG Ta.

A simulation example DTE behavior of CG and UFG pure tantalums were shown in Fig. 9. The number of fragment is correctly prediced by simulation, 7 fragments for CG tantalum and 5 fragments for UFG steel. The simulated total length of CG tantalum and UFG tantalums was $67.43 \mathrm{~mm}$ (DTE elongation $\sim 885 \%$ ) and $46.05 \mathrm{~mm}$ (DTE elongation $\sim 604 \%$ ), respectively. The simulated DTE elonagtion of CG and UFG tantalums are in reasonably good agreement with the experimental one $(\sim 948 \%)$ for CG and $(\sim 648 \%)$ for UFG tantalum repectively.

\section{Summary}

1. A series of dynamic tensile extrusion (DTE) tests, the newly developed mechanical test at high strain rate, was conducted on coarse grained (CG) and ultrafine grained (UFG) OFHC Cu, IF steel, and pure Ta. CG materials exhibited higher DTE elongation than UFG materials.

2. The inferior metal jet stability of UFG materials are caused by its initial higher strength and lack of strain hardenability compared to CG materials.

3. Numerical simulation empoying the mesh adaptivity predicted the fragmentation behavior of CG and UFG materials in terms of DTE elonagtion and the number of fragments. The results of numerical simulation are in reasonsably well agreement with the those of experimental.

\section{References}

[1] Gray III G.T. et al. 2006 Shock Compression of Condense Matter ed by Furnish M.D. et al. (American Institute of Physics) p. 725

[2] Cao F. et al. 2008 Acta Mater. 56, 5804

[3] Escobedo J.P. et al. 2012 Acta Mater. 60, 4379

[4] Farrokh B. and Kahn A.S. 2009 Int. J. Plasticity 25, 715

[5] Q. Wei, T. Jiao, K.T. Ramesh and E. Ma, Scripta Mater. 50, 359 (2004)

[6] D.H. Shin, D.Y. Hwang, Y.J. Oh, K.-T. Park, Metall. Mater. Trans. A 35A, 825 (2004)

[7] A.H. Chokshi, M.A. Meyers, Scripta Metall. Mater. 24, 605 (1990)

[8] Park K.T. et al. 2013 Mater SciEng A 569, 61

[9] LS-DYNA ${ }^{\circledR}$ Keyword User's Manual, 2007 Vol. 1. Version 97 (Livermore Software Technology Corp) 